\title{
Skeletal bone morphology is resistant to the high amplitude seasonal leptin cycle in the Siberian hamster
}

\author{
K Rousseau, Z Atcha, J Denton ${ }^{1}$, F R A Cagampang, A R Ennos, \\ A J Freemont ${ }^{1}$ and $\mathbf{A} S$ I Loudon
}

\begin{abstract}
Faculties of Life Sciences and ${ }^{1}$ Medicine, Stopford Building, University of Manchester, Manchester M13 9PT, UK
(Requests for offprints should be addressed to A S I Loudon, Faculty of Life Sciences, 3.614 Stopford Building, University of Manchester, Oxford Road, Manchester M13 9PT, UK; Email: Andrew.Loudon@manchester.ac.uk)

(F R A Cagampang is now at Centre for Developmental Origins of Health and Disease, University of Southampton, Princess Anne Hospital (F-887), Coxford Road, Southampton SO16 5YA, UK)
\end{abstract}

\begin{abstract}
Recent studies have suggested that the adipocyte-derived hormone, leptin, plays a role in the regulation of metabolism. Here, we tested this hypothesis in the seasonally breeding Siberian hamster, as this species exhibits profound seasonal changes in adiposity and circulating leptin concentrations driven by the annual photoperiodic cycle. Male hamsters were kept in either long (LD) or short (SD) photoperiods. Following exposure to short photoperiods for 8 weeks animals exhibited a significant weight-loss and a 16-fold reduction of serum leptin concentrations. At Week 9, animals in both photoperiods were infused with leptin or PBS via osmotic mini-pump for 14 days. Chronic leptin infusion mimicked LD-like concentrations in SD-

weight and adipose tissue. In LD-housed animals, leptin infusion resulted in a significant elevation of serum concentrations above natural LD-like levels, but had no discernable effect on body weight or overall adiposity. Both bending and compression characteristics and histomorphometric measurements of trabecular bone mass were unaltered by leptin treatment or photoperiod. Our data therefore show that despite a high natural amplitude cycle of leptin, this hormone has no apparent role in the regulation of bone metabolism, and therefore do not support recent propositions that this hormone is an important component in the metabolism of bone tissue. Journal of Endocrinology (2005) 186, 475-479
\end{abstract} housed animals and caused a further decline in body

\section{Introduction}

The adipocyte-derived hormone, leptin, plays an important role in energy homeostasis (Schwartz et al. 2000). It has recently been suggested that in addition to its role in energy metabolism, this hormone also plays a significant role in the development and remodelling of bone. Here, the action of leptin may depend upon its route of administration. Ducy et al. (2000) have reported that obese mice deficient in leptin production ( $o b / o b$ genotype) or devoid of functional leptin receptor expression ( $d b / d b$ genotype) exhibit a markedly elevated bone mass with increased mineralization and bone formation rate compared with wild-type mice. Intracerebroventricular (i.c.v) infusion of leptin in the ob/ob mice was shown to reverse this phenotype (Ducy et al. 2000). This has led to the recent view that in addition to its action on hypothalamic pathways controlling food intake, leptin may also act as a powerful inhibitor of bone formation.

Seasonally breeding mammals commonly exhibit substantial changes in the level of stored fat reserves and circulating leptin concentrations (Rousseau et al. 2003). These adaptations are known to be driven by the seasonal daylength signal, which drives a hypothalamic relay involving the production of a daily pineal melatonin signal. This, in turn, acts on pathways that regulate the set-point for energy expenditure and food intake. As a result, seasonal mammals such as Siberian hamsters exhibit profound photoperiod-driven cycles of adiposity. Exposure to short winter daylength (SD) leads to a loss of up to $40 \%$ body weight, mostly in the form of fat (Steinlechner et al. 1983, Ebling 1994) and a 4-8 fold reduction in circulating leptin concentrations (Rousseau et al. 2003), compared with long photoperiod (LD).

We report experiments in which we tested the hypothesis that chronic exogenous leptin infusions would induce significant changes in bone metabolism. We infused leptin to both SD- and LD-housed animals at a dose designed to mimic natural levels observed on LD photoperiods, and then recorded the impact of these treatments on skeletal morphology. 


\section{Material and Methods}

\section{Animals}

All animal procedures involving leptin infusions were licensed under the Animal (Scientific Procedures) Act of 1986, United Kingdom.

Adult male Siberian hamsters (Phodopus sungorus) from a colony bred at the University of Manchester and derived from animals described previously (Ebling 1994) were kept under controlled conditions (temperature, $21 \pm 1{ }^{\circ} \mathrm{C}$; humidity, 80\%). All animals were individually housed within light-controlled environmental chambers lit by a 70-watt fluorescent white strip (100-400 lux) with continuous dim red light (<1 lux) throughout. Long-day (LD) photoperiods were $16 \mathrm{~h}$ light: $8 \mathrm{~h}$ darkness and short-day (SD) photoperiods were $8 \mathrm{~h}$ light: $16 \mathrm{~h}$ darkness. In the dark phase, a background illumination of dim red light was used ( $<1$ lux).

\section{Leptin infusion protocols}

Recombinant murine leptin (supplied by Amgen Inc., Thousand Oaks, CA, USA) was dissolved in 0.01 M PBS and administered via a 7-day osmotic mini-pump $(100 \mu \mathrm{l}$ capacity; Alzet model 1007D, Charles River Laboratories UK, Kent, UK) to deliver a dose of $15 \mu \mathrm{g} /$ day per animal over a 14-day period. Control animals received PBS vehicle alone. Pumps were implanted subcutaneously in the scapular region by sterile surgical procedure under halothane (Fluothane, AstraZeneca, Cheshire, UK) anesthesia, as described previously (Atcha et al. 2000). The leptin infusions induced a similar serum concentration of leptin to that observed naturally under summer daylengths (circa $15 \mathrm{ng} / \mathrm{ml}$ ) and causes significant fat and weight loss (Atcha et al. 2000).

\section{Experimental protocols}

Twenty-four weight-matched 16-week-old intact male hamsters reared in LD were individually housed and exposed to either LD or SD conditions for an 8-week period, as previously described (Atcha et al. 2000). At Week 9, each animal received an osmotic mini-pump containing either leptin or PBS (six animals per group per treatment). Pumps were replaced after 7 days for a further 1 -week period. On Day 14 of treatment, a single blood sample was taken from each hamster under halothane anaesthesia by cardiac puncture for serum leptin determinations as previously described (Rousseau et al. 2002). Animals were then killed by cervical dislocation and the abdominal fat pads dissected and weighed. At the time of dissection, the carcasses were placed in 100\% ethanol.

\section{Measurement of trabecular bone mass}

During dissection, vertebral tissue was maintained in ethanol and a strip of three thoracic vertebrae (T7 to T9) removed. Tissue was dehydrated in ethanol, delipidised in chloroform and infiltrated in white glycol methacrylate resin (London Resin Co., Theale, Berkshire, $\mathrm{UK})$, then polymerised in an anaerobic, temperature controlled environment. The tissue blocks were then orientated and sectioned coronally in the midpoint of the vertebral bodies. Five $\mu \mathrm{m}$ sections were stained with toluidine blue and mounted, using the standard methodologies employed in the Osteoarticular Pathology Laboratory, University of Manchester (Byers et al. 1999). Histomorphometric measurements were made on the T8 vertebral body using a Leica RMDB microscope linked to a Leica QWin image analyser (Leica, Cambridge, UK). The vertebral body can be considered to be an oblong box attached to its neighbouring vertebrae at each end. The ends are actively growing and therefore possess growth plates. As growth plates are not examined in bone histomorphometry, our measurements excluded both the growth plate and the cortical bone, together known as the tissue area (TA). The amount of bone (trabecular bone area; TBA) was measured in each section and expressed as a percentage of TA $((\mathrm{TBA} / \mathrm{TA}) \times 100)$. Unless otherwise stated, all reagents supplied by WWR International Ltd., Poole, Dorset, UK.

\section{Mechanical tests}

Both bending and compression tests were performed using a universal mechanical testing machine (Instron model 4301, Instron, High Wycombe, UK).

Femora were subjected to three point bending tests. The femur was placed on two supports which were set $10 \mathrm{~mm}$ apart. A pushing probe of radius $20 \mathrm{~mm}$ was attached to the load cell and lowered until it just touched the mid-point of the sample. The crosshead was then lowered at a rate of $10 \mathrm{~mm} \mathrm{~min}^{-1}$, bending the sample until it eventually broke. A computer with an interface to the testing machine was used to produce a graph of force vs displacement, permitting calculation of two mechanical properties of the femur: its bending strength and bending rigidity.

For the compression test, a strip of four lumbar vertebrae (L1 to L4) was removed in ethanol. Before the test, the vertebrae were individually dissected and rehydrated in saline overnight. Each lumbar vertebra was broken in compression along its longitudinal axis at a displacement rate of $5 \mathrm{~mm} / \mathrm{min}$. A load-displacement curve was recorded online and analysed for yield load (i.e. force causing the first damage of the vertebra).

\section{Statistical analysis}

Data were analyzed by $t$-tests, two-way ANOVA or two-way repeated measures of ANOVA followed by post-hoc Student-Newman-Keuls multiple comparison tests, using SigmaStat statistical software (SPSS, Chicago, 
a)

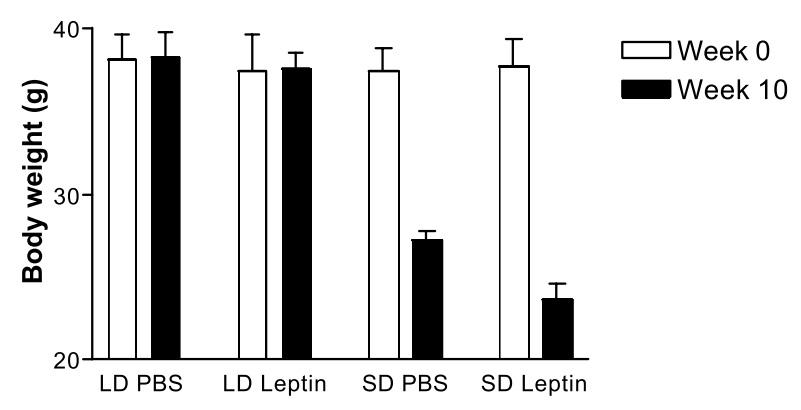

b)

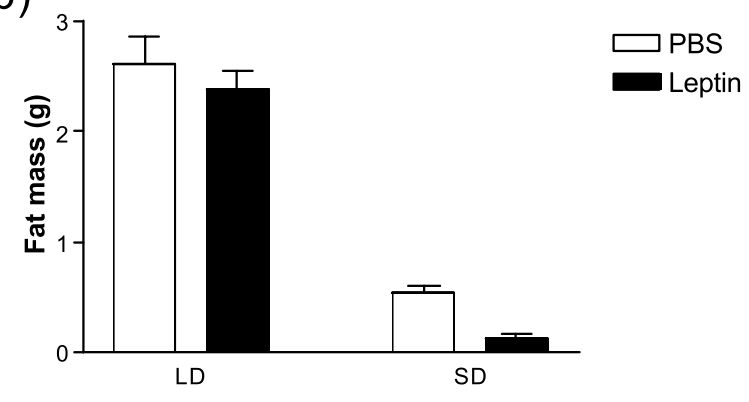

C)

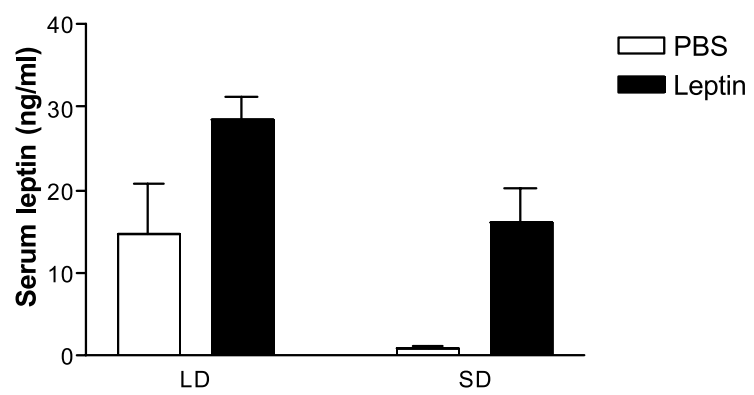

Figure 1 Effect of chronic leptin infusion on body weight (a), fat mass (b) and plasma leptin concentrations (c) in Siberian hamsters. a) shows the body weights measured at the beginning (Week 0; open bars) and at the end (Week 10; closed bars) of the experiment. Week 10 corresponds to the 8 weeks in either LD or SD photoperiods plus the 2 weeks of leptin infusion. Open bars represent the vehicle-treated (PBS) control groups and closed bars represent the leptin-infused groups ( $b$ and $c)$.Values are the means \pm S.E.M.

IL, USA). Results are presented as the means \pm S.E.M. Differences were considered statistically significant at the $P<0 \cdot 05$ level.

\section{Results}

Effect of chronic leptin infusion on body weight, fat mass and serum leptin concentrations

Body weight and fat mass (Fig. 1a and b) were significantly
$(P<0 \cdot 001)$ reduced in SD (body weight $(\mathrm{BW})$ : $27.22 \pm 0.57 \mathrm{~g}$; fat mass: $0.54 \pm 0.05 \mathrm{~g})$ compared with LD (BW: $38 \cdot 22 \pm 1 \cdot 48 \mathrm{~g}$; fat mass: $2.62 \pm 0 \cdot 25 \mathrm{~g}$ ) animals. In SD-housed animals, chronic leptin infusion mimicked LD levels $(15.92 \pm 4.3 \mathrm{ng} / \mathrm{ml}$ SD leptin versus 14.63 $\pm 6.01 \mathrm{ng} / \mathrm{ml}$ LD PBS; Fig. 1c), and caused further body weight $(23.53 \pm 1.06 \mathrm{~g}$ SD leptin versus $27.22 \pm 0.57 \mathrm{~g}$ SD PBS; $P<0.05$; Fig. 1a) and fat $(0 \cdot 12 \pm 0.05 \mathrm{~g} \mathrm{SD}$ leptin versus $0.54 \pm 0.05 \mathrm{~g}$ SD PBS; $P<0.001$; Fig. 1b) losses. Leptin infusion had no effect on LD-housed animals on either body weight $(37.49 \pm 1.02 \mathrm{~g}$ LD leptin versus $38.22 \pm 1.48 \mathrm{~g}$ LD PBS; Fig. 1a) or fat mass (2.39 $\pm 0.16 \mathrm{~g}$ LD leptin versus $2 \cdot 62 \pm 0.25 \mathrm{~g}$ LD PBS; Fig. 1b).

\section{Effect of chronic leptin infusion on bone mass and strength}

Trabecular bone mass (TBA/TA \%) was not significantly altered by photoperiod: $14 \cdot 67 \pm 0.73 \%$ in LD compared with $17 \cdot 27 \pm 2 \cdot 07 \%$ in SD (Fig. 2a). Leptin infusion similarly did not affect trabecular bone mass in both LD $(17 \cdot 20 \pm 2 \cdot 55 \% \mathrm{LD}$ leptin versus $14 \cdot 67 \pm 0 \cdot 73 \% \mathrm{LD}$ PBS $)$ and SD $(16 \cdot 35 \pm 1 \cdot 70 \%$ SD leptin versus $17 \cdot 27 \pm 2 \cdot 07 \%$ SD PBS) (Fig. 2a).

The mechanical properties of cortical bone were assessed by the three point bending test of the femur (Fig. 2b). Bending strength was not affected by photoperiod $\left(31.96 \pm 1.61\right.$ Newton metres $\times 10^{-3}$ in LD versus $32 \cdot 28 \pm 2 \cdot 44$ Newton metres $\times 10^{-3}$ in SD). Leptin infusion did not induce any significant change in bending strength in both LD (32.04 \pm 2.57 Newton metres $\times 10^{-3}$ LD leptin versus $31.96 \pm 1.61$ Newton metres $\times 10^{-3}$ LD PBS) and SD (34.35 $\pm 2 \cdot 74$ Newton metres $\times 10^{-3}$ SD leptin versus $32 \cdot 28 \pm 2 \cdot 44$ Newton metres $\times 10^{-3}$ SD PBS). Similarly, neither leptin infusion nor photoperiod affected bending rigidity (data not shown).

The mechanical properties of trabecular bone were next investigated by a vertebral compression test (Fig. 2c). Vertebra compression strength was not affected by photoperiod $(55 \cdot 6 \pm 3$ Newtons in LD versus $52 \pm 4 \cdot 5$ Newtons in SD). Leptin infusion did not induce any change in compression strength in both $\mathrm{LD}(50 \cdot 10 \pm 2 \cdot 3$ Newtons LD leptin versus $55 \cdot 6 \pm 3$ Newtons LD PBS) and SD $(55 \cdot 3 \pm 2 \cdot 1$ Newtons SD leptin versus $52 \pm 4 \cdot 5$ Newtons SD PBS).

\section{Discussion}

Our data show that despite remarkable natural high amplitude changes in adiposity and circulating leptin concentrations, this was not associated with any significant changes in bone morphology. Our measurements examined both the morphometric and mechanical properties of bone, and neither were significantly altered by 
a)

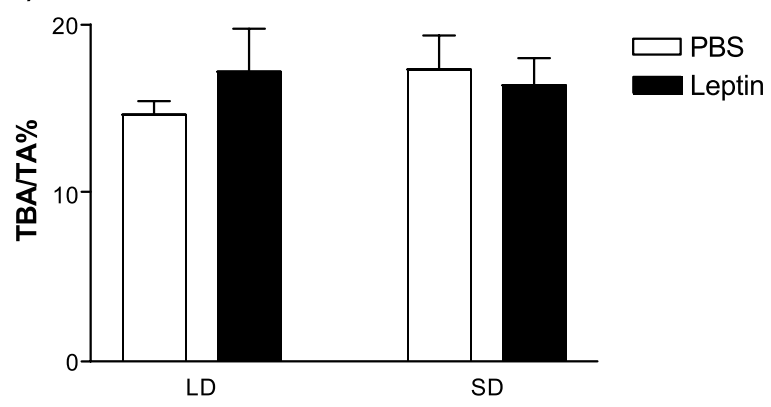

b)

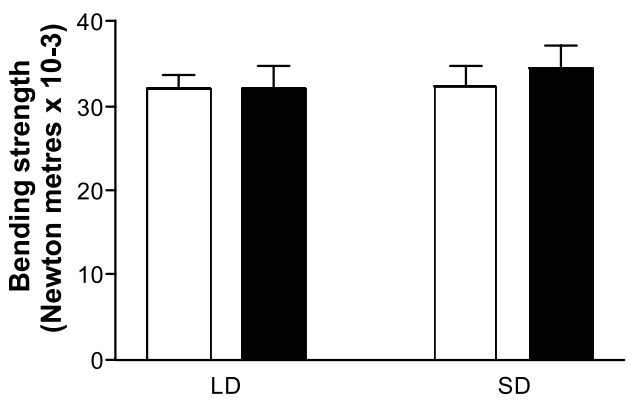

c)

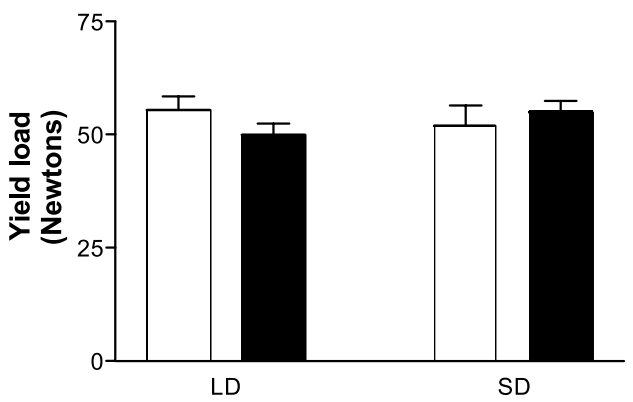

Figure 2 Effect of chronic leptin infusion on bone mass and strength in Siberian hamsters. Trabecular bone mass of thoracic vertebra (a), femur bending strength (b) and compression strength of lumbar vertebra (c) were measured. Open bars represent the vehicle-treated (PBS) control groups and closed bars represent the leptin-infused groups. Values are the means \pm S.E.M.

photoperiod change or chronic leptin infusions designed to mimic maximal long-day concentrations. As a consequence, we conclude that fluctuating leptin concentrations have no significant action on bone metabolism in this species.

Studies of laboratory rodents have recently provided some evidence for a role of leptin in bone re-modelling. Karsenty's laboratory reported that obese rodent models with defective leptin $(o b / o b)$ or lacking a functional leptin receptor $(d b / d b)$ displayed a markedly elevated bone mass (Ducy et al. 2000). When treated with recombinant leptin via a central (i.c.v) route, both obese $o b / o b$ mice and normal mice exhibited significantly decreased bone mass (Ducy et al. 2000, Takeda et al. 2002, Elefteriou et al. 2004). Moreover, studies of two (SAP-leptin and ApoE-leptin) transgenic mouse strains showed that increasing serum leptin levels reduced bone mass (Elefteriou et al. 2004).

In our study, we have used a model species in which there are profound natural changes in leptin and adiposity driven by photoperiod, and have provided a 14-day signal designed to mimic the high levels achieved naturally on long photoperiods. We have shown elsewhere that longday housed hamsters are refractory to these leptin infusion protocols and do not exhibit any significant feeding, reproductive or weight-loss response (Atcha et al. 2000). We have proposed that this resistance to leptin may be mediated at the level of the CNS (Rousseau et al. 2002). Here, our data clearly show that LD-housed animals do not respond to leptin in terms of any of the skeletal parameters measured. Similarly, SD-housed animals, despite differences induced by leptin in terms of metabolic responses, failed to show any significant effect of leptin infusion on bone mass and strength. Thus, our studies clearly indicate that both naturally induced changes and exogenous chronic infusions of this hormone are ineffective in causing skeletal re-modelling. There were no obvious differences in the morphology of vertebral bone in our hamster material compared with laboratory rodents such as mice or rats.

Several laboratories have published contradictory evidence for a role of leptin in bone metabolism. In contrast to studies on mice, obese $f a / f a$ rats, which have a mutated leptin receptor, are reported to have a decreased bone mass (Foldes et al. 1992, Picherit et al. 2003). Models in which leptin has been administered centrally via an i.c.v route have shown a reduced bone mass (Ducy et al. 2000, Takeda et al. 2002, Elefteriou et al. 2004). However, models employing peripheral administration of leptin have been reported to increase bone growth in $o b / o b$ mice (Liu et al. 1997, Steppan et al. 2000) and to reduce the extent of bone loss in ovariectomized rats (Burguera et al. 2001), while systemic administration of leptin to adult male mice has been reported to increase bone strength (Cornish et al. 2002). It has been proposed that the paradoxical differences between central and peripheral effects of leptin on bone re-modelling in laboratory rodents are due to the effects of high peripheral levels leading to a state of central leptin resistance, due perhaps to saturation of receptors (Khosla 2002).

In humans, some in vitro studies clearly demonstrate a local and direct effect of leptin on human osteoblasts. Indeed, expression of leptin and its receptor was shown in primary cultures of normal human osteoblasts (Reseland et al. 2001), while exogenous leptin promoted osteoblastic cell proliferation and differentiation as well as bone mineralization (Reseland et al. 2001, Gordeladze et al. 2002). Nevertheless, other data are inconclusive. In 
cross-sectional studies, although some authors reported a positive association between serum leptin levels and bone mineral density, others failed to find such a relationship or even observed a negative relationship (for a review see Thomas 2004). Those few interventional studies conducted in humans have also produced conflicting outcomes. For instance, subcutaneous leptin therapy for up to four years in three morbidly obese children congenitally deficient in leptin did restore bone mass to normal age-related development (Farooqi et al. 2002). However, two groups (Simha et al. 2002, Moran et al. 2004) have reported that in patients with generalized lipodystrophy, chronic exogenous leptin administration had no impact on bone mass density.

In conclusion, our studies do not support recent investigations in rodents that have reported the action of leptin on bone metabolism. Using a leptin infusion model, our data suggest that in wild seasonally breeding animals adaptations may exist to ensure that skeletal characteristics are able to resist seasonal changes in energy metabolism and circulating leptin concentrations. Our data therefore cast some doubt on the proposition that leptin may play an important general role in the metabolism of bone in species other than laboratory rats and mice.

\section{Acknowledgements}

The authors thank Gary Dawes for care and attention to the animals, Alexander Raby for assistance in bone bending studies, Margery Nicholson and colleagues at Amgen, Dr Martin Klingenspor, Dr Nigel Brooks and the BBSRC for financial support. The authors declare that there is no conflict of interest that would prejudice the impartiality of this scientific work.

\section{References}

Atcha Z, Cagampang FRA, Stirland JA, Morris ID, Brooks AN, Ebling FJ, Klingenspor M \& Loudon ASI 2000 Leptin acts on metabolism in a photoperiod-dependent manner, but has no effect on reproductive function in the seasonally breeding Siberian hamster (Phodopus sungorus). Endocrinology 141 4128-4135.

Burguera B, Hofbauer LC, Thomas T, Gori F, Evans GL, Khosla S, Riggs BL \& Turner RT 2001 Leptin reduces ovariectomy-induced bone loss in rats. Endocrinology 142 3546-3553.

Byers RJ, Denton J, Hoyland JA \& Freemont AJ 1999 Differential patterns of altered bone formation in different bone compartments in established osteoporosis. Journal of Clinical Pathology 52 23-28.

Cornish J, Callon KE, Bava U, Lin C, Naot D, Hill BL, Grey AB, Broom N, Myers DE, Nicholson GC \& Reid IR 2002 Leptin directly regulates bone cell function in vitro and reduces bone fragility in vivo. Journal of Endocrinology 175 405-415.

Ducy P, Amling M, Takeda S, Priemel M, Schilling AF, Beil FT, Shen J, Vinson C, Rueger JM \& Karsenty G 2000 Leptin inhibits bone formation through a hypothalamic relay: a central control of bone mass. Cell 100 197-207.

Ebling FJ 1994 Photoperiodic differences during development in the dwarf hamsters Phodopus sungorus and Phodopus campbelli. General and Comparative Endocrinology 95 475-482.
Elefteriou F, Takeda S, Ebihara K, Magre J, Patano N, Kim CA, Ogawa Y, Liu X, Ware SM, Craigen WJ, Robert JJ, Vinson C, Nakao K, Capeau J \& Karsenty G 2004 Serum leptin level is a regulator of bone mass. PNAS 101 3258-3263.

Farooqi IS, Matarese G, Lord GM, Keogh JM, Lawrence E, Agwu C, Sanna V, Jebb SA, Perna F, Fontana S, Lechler RI, DePaoli AM \& O'Rahilly S 2002 Beneficial effects of leptin on obesity, T cell hyporesponsiveness, and neuroendocrine/metabolic dysfunction of human congenital leptin deficiency. Journal of Clinical Investigation 110 1093-1103.

Foldes J, Shih MS \& Levy J 1992 Bone structure and calcium metabolism in obese Zucker rats. International Journal of Obesity and Related Metabolic Disorders 16 95-102.

Gordeladze JO, Drevon CA, Syversen U \& Reseland JE 2002 Leptin stimulates human osteoblastic cell proliferation, de novo collagen synthesis, and mineralization: impact on differentiation markers, apoptosis, and osteoclastic signaling. Journal of Cellular Biochemistry 85 825-836.

Khosla S 2002 Editorial: Leptin - Central or peripheral to the regulation of bone metabolism? Endocrinology 143 4161-4164.

Liu C, Grossmann A, Bain S, Strachan M, Puerner C, Bailey C, Humes J, Lenox J, Yamamoto G, Sprugel K, Kujiper J, Weigle S, Durnam D \& Moore E 1997 Leptin stimulates cortical bone formation in obese $(o b / o b)$ mice. Journal of Bone and Mineral Research 12 S115.

Moran SA, Pattern N, Young JR, Cochran E, Sebring N, Reynolds J, Premkumar A, Depaoli AM, Skarulis MC, Oral EA \& Gorden P 2004 Changes in body composition in patients with severe lipodystrophy after leptin replacement therapy. Metabolism $\mathbf{5 3}$ 513-519.

Picherit C, Horcajada MN, Mathey J, Chanteranne B, Puel C, Lebecque P, Davicco MJ, Coxam V \& Barlet JP 2003 Isoflavone consumption does not increase the bone mass in osteopenic obese female Zucker rats. Annals of Nutrition and Metabolism 47 70-77.

Reseland JE, Syversen U, Bakke I, Qvigstad G, Eide LG, Hjertner O, Gordeladze JO \& Drevon CA 2001 Leptin is expressed in and secreted from primary cultures of human osteoblasts and promotes bone mineralization. Journal of Bone and Mineral Research 16 1426-1433.

Rousseau K, Atcha Z, Cagampang FRA, Le Rouzic P, Stirland JA, Ivanov TR, Ebling FJP, Klingenspor M \& Loudon ASI 2002 Photoperiodic regulation of leptin resistance in the seasonally breeding Siberian hamster (Phodopus sungorus). Endocrinology 143 3083-3095.

Rousseau K, Atcha Z \& Loudon ASI 2003 Leptin and seasonal mammals. Journal of Neuroendocrinology 15 409-414.

Schwartz MW, Woods SC, Porte Jr D, Seeley RJ \& Baskin DG 2000 Central nervous system control of food intake. Nature 404 661-671.

Simha V, Zerwekh JE, Sakhaee K \& Garg A 2002 Effect of subcutaneous leptin replacement therapy on bone metabolism in patients with generalized lipodystrophy. Journal of Clinical Endocrinology and Metabolism 87 4942-4945.

Steinlechner S, Heldmaier G \& Becker H 1983 The seasonal cycle of body weight in the Djungarian hamster: photoperiodic control and the influence of starvation and melatonin. Oecologia 60 401-405.

Steppan CM, Crawford DT, Chidsey-Frink KL, Ke H \& Swick AG 2000 Leptin is a potent stimulator of bone growth in $o b / o b$ mice. Regulatory Peptides 92 73-78.

Takeda S, Elefteriou F, Levasseur R, Liu X, Zhao L, Parker KL, Armstrong D, Ducy P \& Karsenty G 2002 Leptin regulates bone formation via the sympathetic nervous system. Cell 111 305-317.

Thomas T 2004 The complex effects of leptin on bone metabolism through multiple pathways. Current Opinion in Pharmacology 4 295-300.

Received 1 March 2005

Accepted 28 June 2005 